\title{
Prevalência de leptospirose em caprinos leiteiros do semiárido paraibano ${ }^{1}$
}

\author{
Severino S.S. Higino², Clebert J. Alves², Carolina S.A.B. Santos ${ }^{3}$, Sílvio A. Vasconcellos ${ }^{3}$, \\ Maria L.C.R. Silva ${ }^{2}$, Arthur W.L. Brasil ${ }^{2}$, Carla L.R.M. Pimenta ${ }^{2}$ e Sérgio S. Azevedo ${ }^{2^{*}}$
}

\begin{abstract}
Higino S.S.S., Alves C.J., Santos C.S.A.B., Vasconcellos S.A., Silva M.L.C.R., Brasil A.W.L., Pimenta C.L.R.M. \& Azevedo S.S. 2012. [Prevalence of leptospirosis in dairy goats in the semiarid region of Paraíba state.] Prevalência de leptospirose em caprinos leiteiros do semiárido paraibano. Pesquisa Veterinária Brasileira 32(3):199-203. Universidade Federal de Campina Grande, Centro de Saúde e Tecnologia Rural, Unidade Acadêmica de Medicina Veterinária, Av. Universitária s/n, Patos, PB 58700-970, Brazil. E-mail: sergio.azevedo@pq.cnpq.br

This study aimed to determine the prevalence of positive dairy goat herds and seropositive animals for leptospirosis in the semiarid region of Paraíba state, Brazil. Sampling was designed to determine the prevalence of positive herds (foci) and seropositive animals for leptospirosis. A total of 975 serum samples were collected from goats of 110 dairy herds in the Monteiro municipality, Western Cariri microregion of Paraiba. For the serological diagnosis of leptospirosis the microscopic agglutination test (MAT) was carried out using 24 Leptospira spp. serovars as antigens. A herd was considered positive when existed at least one seropositive animal. The prevalence of positive herds and seropositive animals were $43.6 \%(95 \% \mathrm{CI}=34.2-53.4 \%)$ and $8.7 \%(95 \% \mathrm{CI}=58.7-12.9 \%)$ respectively. The most frequent serovars in goats were Autumnalis $(1.74 \%$; $95 \% \mathrm{CI}=0.97-3.09 \%)$, Sentot $(1.71 \%$; $95 \% \mathrm{CI}=0.82-3.52 \%)$ and Whitcomb $(1.39 \% ; 95 \% \mathrm{CI}=0.65-2.93 \%)$, and by herd, serovars Autumnalis (10.9\%; 95\% CI = 5.8-18.3\%), Whitcomb (8.2\%; 95\% CI = 3.8-15.0\%) and Sentot and Patoc $(7.3 \% ; 95 \% \mathrm{CI}=3.2-13.8 \%)$ were the most frequent. It is suggested that leptospirosis is spread in goats in the region, and that there is need to implement control and preventive measures in order to reduce infection and economic losses, and to avoid possible transmission of infection to humans.
\end{abstract}

INDEX TERMS: Leptospira spp., small ruminants, dairy goats, epidemiology.

RESUMO.- Este trabalho teve como objetivos determinar a prevalência de propriedades de caprinos leiteiros positivas e de animais soropositivos para leptospirose no semiárido paraibano. A amostragem foi delineada para a determinação da prevalência de propriedades positivas (focos) e de animais soropositivos para a infecção por Leptospira spp. No total, foram colhidas amostras de sangue de 975 ani-

\footnotetext{
${ }^{1}$ Recebido em 2 de setembro de 2011.

Aceito para publicação em 25 de outubro de 2011.

${ }^{2}$ Universidade Federal de Campina Grande, Centro de Saúde e Tecnologia Rural, Unidade Acadêmica de Medicina Veterinária, Avenida Universitária s/n, Patos, PB 58700-970, Brasil. *Autor para correspondência: sergio.azevedo@pq.cnpq.br

${ }^{3}$ Departamento de Medicina Veterinária Preventiva e Saúde Animal, Faculdade de Medicina Veterinária e Zootecnia (FMVZ), Universidade de São Paulo (USP), Cidade Universitária, Av. Prof. Dr. Orlando Marques de Paiva 87, São Paulo, SP 05508-270, Brasil.
}

mais procedentes de 110 propriedades leiteiras localizadas no Munícipio de Monteiro, microrregião do Cariri Ocidental, Estado da Paraíba. Para o diagnóstico da infecção por Leptospira spp., foi utilizado o teste de soroaglutinação microscópica (SAM), utilizando como antígenos 24 sorovares. Uma propriedade foi considerada foco quando apresentou pelo menos um animal soropositivo. As prevalências de propriedades positivas e de animais soropositivos foram de $43,6 \%$ (IC $95 \%=34,2-53,4 \%$ ) e de $8,7 \%$ (IC $95 \%=$ $5,7-12,9 \%)$, respectivamente. Nos animais, os sorovares mais frequentes foram Autumnalis (1,74\%; IC 95\% $=0,97$ $3,09 \%)$, Sentot (1,71\%; IC 95\% = 0,82-3,52\%) e Whitcomb $(1,39 \%$; IC $95 \%=0,65-2,93 \%)$, e nas propriedades, os sorovares Autumnalis $(10,9 \%$; IC $95 \%=5,8-18,3 \%)$, Whitcomb $(8,2 \%$; IC $95 \%=3,8-15,0 \%)$ e Sentot e Patoc $(7,3 \%$; IC $95 \%=3,2-13,8 \%$ ) foram os mais frequentes. Sugere-se que a infecção está distribuída em caprinos da região, e 
que há necessidade de implantação de medidas de controle e prevenção, com o intuito de reduzir a ocorrência da infecção e, consequentemente, diminuir perdas econômicas ocasionadas e bloquear a possível transmissão do agente aos seres humanos.

TERMOS DE INDEXAÇÃO: Leptospira spp., pequenos ruminantes, epidemiologia.

\section{INTRODUÇÃO}

A exploração de cabras leiteiras é uma atividade econômica em ascensão no Brasil (Cordeiro 1998). A despeito do grande número de caprinos, aproximadamente 12 milhões, o Brasil ocupa apenas o $18^{\circ}$ lugar no ranking de produção de leite de cabra (Lilenbaum et al. 2008), devido principalmente à baixa produtividade por animal. Dentre outros fatores, as doenças infecciosas, tais como a leptospirose, podem contribuir para este problema, levando a uma diminuição da produção de leite (Cordeiro 1998, Faine et al. 1999).

Zoonose mundialmente distribuída, a leptospirose é particularmente prevalente nas Américas e considerada endêmica na América Latina e no Caribe, com impacto na economia agropecuária. A ocorrência de leptospirose está estreitamente vinculada aos fatores ambientais, que podem dar lugar a um foco de infecção, cuja amplitude está na dependência de condições favoráveis, das características do habitat e da presença de animais silvestres (Alves et al. 1996, Genovez et al. 2006). Hospedeiros adaptados ou de manutenção são altamente susceptíveis, cujo ciclo de infecção é perpetuado dentro da mesma espécie, usualmente por transmissão direta. Os sovares adaptados aos hospedeiros naturais favorecem a sua manutenção no meio ambiente, podendo atingir, por transmissão indireta, os hospedeiros incidentais, que são infectados de forma acidental, geralmente por espécie diferente. 0 ser humano se comporta na maioria das vezes como hospedeiro incidental, pois raramente se constitui em transmissor da infecção (Faine et al. 1999).

Embora tenha nos caprinos frequência reduzida, a disseminação de leptospiras entre eles é um fato real e crescente, sendo agravado em propriedades que adotam atividades consorciadas com outras espécies animais (Langoni et al. 1995). A despeito dos caprinos serem considerados menos susceptíveis à infecção por Leptospira spp. (Leon-Vizcaino et al. 1987), alguns relatos no Brasil demonstraram soropositividades variando de $24 \%$ a $76 \%$ (Cunha et al. 1999).

Na infecção aguda dos caprinos, observa-se anorexia, dificuldade respiratória, anemia hemolítica, icterícia, urina de cor vermelho-escura e febre. A forma crônica, que ocorre com mais frequência, é caracterizada por baixa fertilidade, mortalidade neonatal, abortamentos e diminuição da produção de leite, levando a perdas econômicas importantes (Cunha et al. 1999).

Considerando a importância da caprinocultura para a região Nordeste do Brasil, particularmente para a microrregião do Cariri Ocidental paraibano, as perdas econômicas ocasionadas pela leptospirose, a possibilidade de trans- missão de leptospiras para os seres humanos, bem como a escassez de informações acerca dos sorovares de Leptospira spp. nos rebanhos caprinos paraibanos, o objetivo do presente trabalho foi determinar a prevalência de propriedades positivas (focos) e animais soropositivos para a infecção por Leptospira spp. em propriedades de caprinos leiteiros no semiárido paraibano.

\section{MATERIAL E MÉTODOS}

\section{Animais e amostragem}

Foram utilizadas fêmeas caprinas adultas provenientes de propriedades localizadas no Município de Monteiro, microrregião do Cariri Ocidental, Estado da Paraíba. A amostragem foi delineada para a determinação da prevalência de propriedades positivas (focos) e de animais soropositivos para a infecção por Leptospira spp., e foi realizada em duas etapas: (1) uma seleção aleatória de um número pré-estabelecido de propriedades (unidades primárias); (2) dentro das unidades primárias, foi amostrado, aleatoriamente, um número pré-estabelecido de caprinos (unidades secundárias).

Para o cálculo do número de unidades primárias a serem amostradas, foram considerados os seguintes parâmetros: (a) prevalência esperada; (b) erro absoluto; e (c) nível de confiança, de acordo com a fórmula para amostras aleatórias simples (Thrusfield 1995, Noordhuizen et al. 1997):

$$
n=\frac{Z^{2} \times P(1-P)}{d^{2}}
$$

Onde:

$n=$ número de propriedades amostradas

$Z$ = valor da distribuição normal para o nível de confiança de $95 \%$

$P=$ prevalência esperada de $20,8 \%$ (Lilenbaum et al. 2008)

$d=$ erro absoluto de $5 \%$

Para o ajuste para populações finitas, foi utilizada a seguinte fórmula (Thrusfield 1995):

$$
n_{a j u s}=\frac{N \times n}{N+n}
$$

Onde:

$n_{\text {ajus }}=$ tamanho da amostra ajustado

$N=$ tamanho da população total

$n=$ tamanho inicial da amostra

De acordo com o Centro de Desenvolvimento Integrado da Caprinovinocultura (CENDOV), atualmente há 180 propriedades de exploração de cabras leiteiras cadastradas. Com base nesses dados, o número de unidades primárias a serem visitadas é de 105. Por motivo de segurança, foram utilizadas 110 propriedades.

O número de animais testados para um rebanho ser classificado como positivo ou negativo foi calculado com base no valor de sensibilidade e especificidade agregadas (Martin et al. 1992, Jordan 1996). Dessa forma, o cálculo do número de unidades secundárias foi realizado com o programa Herdacc versão 3.0, de modo a ser obtido um valor de sensibilidade e especificidade agregadas de pelo menos 90\% (Martin et al. 1992, Jordan 1996), utilizando os seguintes parâmetros:

a) Sensibilidade e especificidade do teste, em nível individual, de $80 \%$ e $100 \%$, respectivamente (Vasconcellos et al. 1990).

b) Tamanho do rebanho. 
c) Ponto de corte 1, ou seja, número mínimo de animais positivos para classificar o rebanho como foco.

Após várias simulações no programa Herdacc versão 3.0, optou-se pelos seguintes tamanhos amostrais:

- Propriedades com até 100 fêmeas adultas => foram amostrados 12 animais.

- Propriedades com mais de 100 fêmeas adultas => foram amostrados 13 animais.

- Propriedades com até 12 fêmeas adultas => foram amostrados todos os animais.

No total, foram amostradas 975 fêmeas caprinas adultas procedentes de 110 propriedades, no período de março a julho de 2009.

\section{Diagnóstico sorológico da infecção por Leptospira spp.}

O diagnóstico sorológico da leptospirose foi realizado pela técnica de soroaglutinação microscópica (SAM) (Galton et al. 1965, Cole et al. 1973), com uma coleção de antígenos vivos composta por 22 sorovares patogênicos e dois saprófitos: Australis, Bratislava, Autumnalis, Butembo, Castellonis, Bataviae, Canicola, Whitcombi, Cynopteri, Grippotyphosa, Hebdomadis, Copenhageni, Icterohaemorrhagiae, Javanica, Panamá, Pomona, Pyrogenes, Hardjo, Wolffi, Shermani, Tarassovi, Andamana, Patoc e Sentot.

Os soros foram triados na diluição de 1:100, e aqueles que apresentaram $50 \%$ ou mais de aglutinação foram titulados pelo exame de uma série de diluições geométricas de razão dois. 0 título do soro foi a recíproca da maior diluição que apresentou resultado positivo. Os antígenos foram examinados ao microscópio de campo escuro, previamente aos testes, a fim de verificar a mobilidade e a presença de auto-aglutinação ou de contaminantes.

Uma propriedade foi considerada positiva (foco) quando apresentou pelo menos um animal soropositivo. No animal, o provável sorovar infectante foi o que apresentou o maior título, e na propriedade foi o que apresentou maior título e/ou número de reações positivas. Os animais que apresentaram dois ou mais sorovares com títulos idênticos foram considerados positivos, porém desconsiderados para o cálculo do sorovar mais frequente.

\section{Confecção de mapas georreferenciados}

As coordenadas geográficas das propriedades amostradas obtidas com um aparelho de GPS (Global Position System) e os limites geográficos da região foram plotados em um mapa georreferenciado digital do Estado da Paraíba, baseado no mapa da malha municipal do Brasil de 2001, obtido junto ao Instituto Brasileiro de Geografia e Estatística (IBGE). As atividades de plotagem e tratamento dos mapas digitais foram efetuadas com o programa ArcGIS versão 9.1. Foram construídos dois mapas: um com a distribuição geográfica das propriedades positivas e negativas, e outro com a distribuição dos sorovares mais prevalentes por propriedade.

\section{Cálculo das prevalências}

Para o cálculo da prevalência de focos, foi considerado o delineamento amostral de uma amostra aleatória simples, utilizando os parâmetros: (a) número de focos; e (b) número de propriedades amostradas. 0 delineamento amostral para o cálculo da prevalência de animais soropositivos empregou uma amostra de grupo (cluster) em dois estágios (Thrusfield 1995), onde cada propriedade foi considerada um grupo. Os parâmetros utilizados foram: (a) condição do animal (soropositivo ou soronegativo); (b) código do rebanho (para identificar cada grupo); e (c) peso estatístico. 0 peso estatístico foi calculado com a seguinte fórmula (Dean 1994):

$$
\begin{aligned}
& \text { Peso }=\frac{\text { matrizes caprinasna região }}{\text { matrizes caprinas nas pro- }} \times \frac{\text { matrizes caprinasna propriedade }}{\text { matrizes caprinas amostradas na }} \\
& \text { priedades amostradas } \\
& \text { propriedade }
\end{aligned}
$$

\section{RESULTADOS E DISCUSSÃO}

Das 110 propriedades amostradas, 48 propriedades apresentaram pelo menos um animal reagente na SAM para qualquer sorovar, o que resultou em uma prevalência de focos de 43,6\% (IC 95\% = 34,2-53,4\%). No Quadro 1 é apresentado o número de propriedades positivas na SAM aplicada à leptospirose de acord o com os sorovares. 0 sorovar Autumnalis foi o mais frequente, com 12 propriedades positivas $(10,9 \%)$, seguido do Whitcombi com nove $(8,2 \%)$, Sentot e Patoc com oito propriedades cada (7,3\%), Butembo com cinco $(4,5 \%)$, e Andamana com quatro $(3,6 \%)$ propriedades positivas. Na Figura 1 é apresentada a distribuição espacial dos focos de leptospirose e, na Figura 2, a distribuição espacial dos sorovares mais prevalentes nos focos.

Dos 975 caprinos analisados, 98 foram positivos pela SAM, obtendo-se uma prevalência de animais soropositivos de 8,7\% (IC 95\% = 5,7-12,9\%; efeito do desenho = 4,23). No Quadro 2 é apresentado o número de animais reagentes na SAM de acordo com os sorovares com as respectivas prevalências. O sorovar Autumnalis foi o mais frequente, com 25 soros reagentes $(1,74 \%)$, seguido de Sentot com 19 soros (1,71\%), Whitcombi com 14 (1,39\%), Andamana

Quadro 1. Sorovares de Leptospira spp. prevalentes em propriedades de caprinos leiteiros no Município de Monteiro, semiárido do Estado da Paraíba, de março a julho de 2009

\begin{tabular}{lccc}
\hline \multicolumn{1}{c}{ Sorovar } & $\begin{array}{c}\text { Proporção de propriedades Prevalência (\%) } \\
\text { positivas }\end{array}$ & IC 95\% (\%) \\
\hline Autumnalis & $12 / 110$ & $10,9 \%$ & $5,8-18,3$ \\
Whitcombi & $9 / 110$ & $8,2 \%$ & $3,8-15,0$ \\
Sentot & $8 / 110$ & $7,3 \%$ & $3,2-13,8$ \\
Patoc & $8 / 110$ & $7,3 \%$ & $3,2-13,8$ \\
Butembo & $5 / 110$ & $4,5 \%$ & $1,5-10,3$ \\
Andamana & $4 / 110$ & $3,6 \%$ & $1,0-9,0$
\end{tabular}

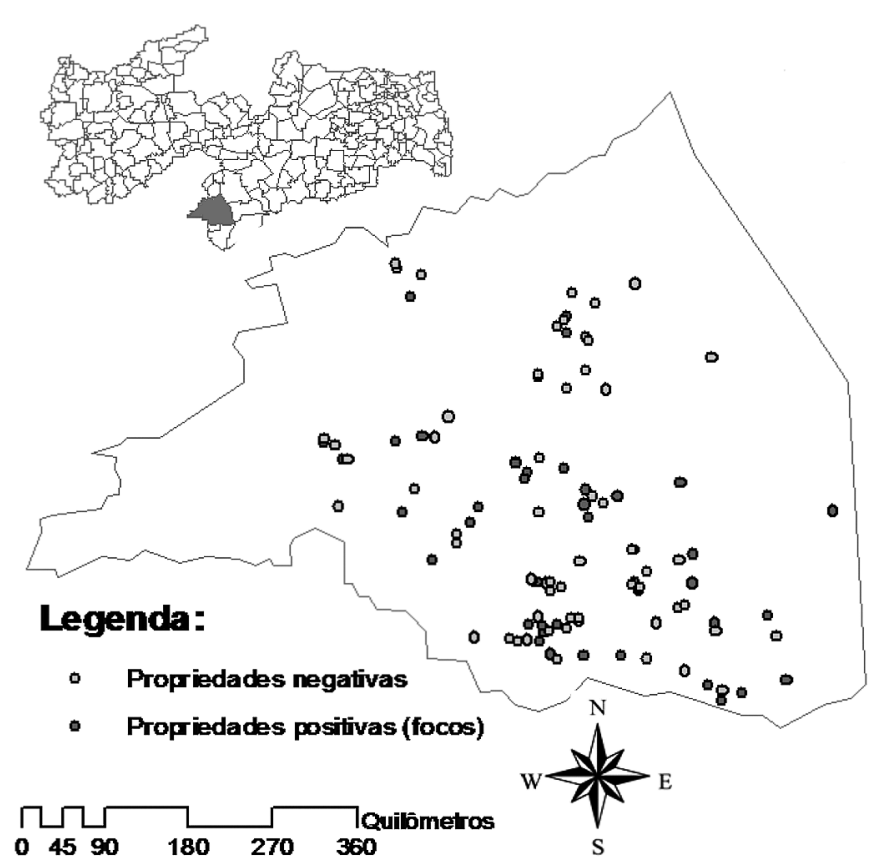

Fig.1. Distribuição espacial dos focos de leptospirose em propriedades de caprinos leiteiros no Município de Monteiro, semiárido do Estado da Paraíba, de março a julho de 2009. 


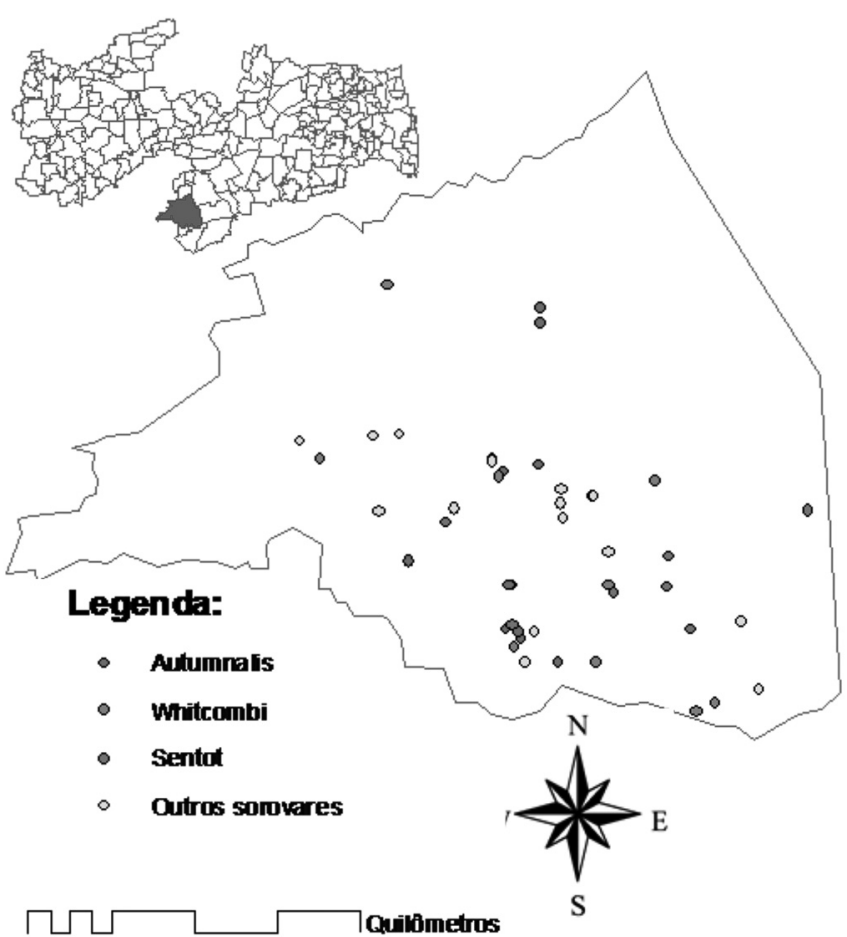

D 4500 180 270 360

Fig.2. Distribuição espacial dos sorovares de Leptospira spp. prevalentes em propriedades de caprinos leiteiros no Município de Monteiro, semiárido do Estado da Paraíba, de março a julho de 2009.

Quadro 2. Sorovares de Leptospira spp. prevalentes em caprinos leiteiros reagentes em relação ao total de animais pela técnica de soroaglutinação microscópica aplicada à leptospirose no Município de Monteiro, semiárido do Estado da Paraíba, de março a julho de 2009

\begin{tabular}{lcccc}
\hline \multicolumn{1}{c}{ Sorovar } & $\begin{array}{c}\text { Proporção de } \\
\text { reagentes }\end{array}$ & $\begin{array}{c}\text { Prevalência } \\
(\%)\end{array}$ & $\begin{array}{c}\text { IC 95\% } \\
(\%)\end{array}$ & $\begin{array}{c}\text { Efeito do } \\
\text { desenho }\end{array}$ \\
\hline Autumnalis & $25 / 975$ & $1,74 \%$ & $0,97-3,09$ & 1,62 \\
Sentot & $19 / 975$ & $1,71 \%$ & $0,82-3,52$ & 2,54 \\
Whitcombi & $14 / 975$ & $1,39 \%$ & $0,65-2,93$ & 2,18 \\
Andamana & $11 / 975$ & $1,31 \%$ & $0,52-3,29$ & 3,11 \\
Patoc & $13 / 975$ & $1,29 \%$ & $0,57-2,89$ & 2,36 \\
Butembo & $7 / 975$ & $0,53 \%$ & $0,19-1,46$ & 1,50 \\
Castellonis & $1 / 975$ & $0,05 \%$ & $0,01-0,37$ & 0,54 \\
Bratislava & $1 / 975$ & $0,04 \%$ & $0,01-0,30$ & 0,45 \\
Pyrogenes & $1 / 975$ & $0,03 \%$ & $0,00-0,20$ & 0,30
\end{tabular}

com 11 (1,31\%), Patoc com 13 (1,29\%), Butembo com sete $(0,53 \%)$, Castellonis, Bratislava e Pyrogenes com um soro $(0,05 \%, 0,04 \%$ e $0,03 \%$, respectivamente).

Lilenbaum et al. (2008) avaliaram 248 caprinos oriundos de 13 propriedades no Rio de Janeiro, e encontraram 52 animais positivos, com uma soroprevalência de $20,8 \%$. Silva et al. (2006) analisaram 450 amostras de soro caprino de 45 rebanhos da microrregião do Cariri Paraibano, e encontraram $59(13,1 \%)$ amostras positivas. Fávero et al. (2002) avaliaram 1262 caprinos no Estado da Paraíba em estudo retrospectivo, e encontraram $65(5,1 \%)$ animais positivos. Schimidt et al. (2002) examinaram 354 soros de caprinos leiteiros de 15 municípios do Rio Grande do Sul, e identificaram $56(3,4 \%)$ animais positivos. A diferença entre os resultados obtidos nos vários estudos de prevalência da leptospirose em caprinos pode estar relacionada à va- riedade de fatores que influenciam na ocorrência da doença, como as espécies animais de contato, manejo utilizado, os sorovares existentes na região, as condições climáticas e ambientais e das oportunidades de infecção direta ou indireta. Tendo em vista que os animais amostrados no presente estudo são animais leiteiros, o tipo de manejo utilizado, a estrutura da produção, as medidas higiênico-sanitárias entre outros, são fatores que contribuem para diminuição da prevalência da leptospirose.

O uso de amostragem por conveniência em estudos de ocorrência de doenças infecciosas é muito comum e possibilita o levantamento de informações importantes, entretanto, inferências epidemiológicas não devem ser feitas com base nesse procedimento tendo em vista a ocorrência de vieses. 0 presente estudo é o primeiro, conduzido com base em delineamento amostral adequado, sobre a ocorrência e distribuição de leptospirose em caprinos leiteiros do semiárido da Paraíba.

Uma característica que exerce influência no tamanho da amostra é o desenho amostral, isto é, de que forma as unidades amostrais são selecionadas. Na prevalência de animais soropositivos para a leptospirose, o desenho amostral empregado foi a amostragem de grupo em dois estágios. Do ponto de vista prático e operacional, este tipo de desenho é o mais simples, no entanto, a imprecisão na estimativa da prevalência é maior do que quando se usa uma amostragem simples aleatória. Exemplo desta imprecisão foi o efeito do desenho no cálculo da prevalência de animais soropositivos, cujo valor foi de 4,23. 0 efeito do desenho pode ser definido como sendo a razão entre as imprecisões associadas à estimativa de um parâmetro sob dois desenhos amostrais (Luiz \& Magnanini 2005), ou seja, na estimativa da prevalência de fêmeas soropositivas, é a razão entre a variância da estimativa na amostragem de grupo e a variância da estimativa assumindo-se que os dados viessem de uma amostra simples aleatória. Tomando-se como base o efeito do desenho de 4,23, significa dizer que a variância foi estimada como sendo 4,23 vezes a variância para uma amostra simples aleatória, ou seja, o intervalo de confiança obtido é 4,23 vezes o intervalo de confiança caso a amostragem utilizada fosse a simples aleatória. Assim, o efeito do desenho funciona como um "preço" a ser pago pela facilitação da tarefa ao se investigar apenas os grupos sorteados, aumentando a imprecisão na estimativa da prevalência devido às possíveis correlações das unidades amostrais dentro e entre os grupos.

Nesta pesquisa o sorovar mais prevalente foi o Autumnalis tanto para propriedades quanto para animais, resultados igualmente encontrados por Silva et al. (2006), que analisaram 450 amostras de soro caprino de 45 rebanhos da microrregião do Cariri Paraibano e obtiveram 44 soros reagentes para o sorovar Autumnalis (74,6\%). 0 sorovar Autumnalis possui como principais reservatórios os roedores, indicando a importância desses animais como prováveis fontes de infecção para os caprinos, bem como a necessidade de intensificação do controle dos mesmos.

Estudos conduzidos em caprinos no semiárido nordestino apontaram predominância de reações para o sorovar Autumnalis. Araújo Neto (2005) utilizou 100 ovelhas abatidas no matadouro público de Patos, $\mathrm{PB}$, para o isolamento 
da bactéria a partir do trato genital, realizando paralelamente a sorologia, obtendo $9 \%$ de soropositividade para o agente, e 44,4\% de frequência para o sorovar Autumnalis. Higino (2007) realizou a sorologia de 80 ovinos e obteve $7,5 \%$ de positividade, com $83,3 \%$ de frequência para o sorovar Autumnalis. Esses estudos, bem como os resultados do presente trabalho, levantam a hipótese dos caprinos e ovinos como fontes de infecção do sorovar Autumnalis na região e reforçam o risco de transmissão para os seres humanos e para outras espécies.

A ocorrência do sorovar Autumnalis causa preocupação, pois não existe imunidade cruzada entre os diferentes sorovares, e no mercado as vacinas são compostas, basicamente, pelos sorovares Canicola, Icterohaemorrhagiae, Pomona, Grippotyphosa, Hardjo, Tarassovi, Andamana, Wolffii e Bataviae, o que alerta para a importância da produção de novas vacinas contra a leptospirose e a necessidade de inclusão de novos sorovares, visando à elaboração de vacinas mais efetivas e de imunidade mais duradoura.

O sorovar Sentot foi apontado como o segundo mais frequente nos animais e terceiro nas propriedades. Herrmann et al. (2004) testaram 1360 amostras de soros ovinos nas Mesorregiões Sudeste e Sudoeste do Estado do Rio Grande do Sul e encontraram 466 animais reagentes, e pela primeira vez no Brasil relataram a presença do sorovar Sentot em animais domésticos, com o agravante de ser o segundo sorovar mais frequente (16,8\%). Corrêa et al. (1964) relataram dois casos do sorovar Sentot em pacientes humanos no Estado de São Paulo, os primeiros a serem registrados no Brasil, e referiram que este sorovar é descrito como causador de doença clínica em seres humanos. Os achados destes estudos e os resultados do presente trabalho ressaltam a importância do sorovar Sentot em casos de leptospirose humana, e o possível risco de transmissão entre seres humanos e caprinos.

\section{CONCLUSÃO}

Observou-se que a infecção por Leptospira spp. é distribuída em caprinos da região estudada e que para reduzir sua ocorrência e, consequentemente, diminuir perdas econômicas ocasionadas e bloquear a possível transmissão do agente aos seres humanos, se faz necessária a implantação de medidas de controle e prevenção.

Agradecimentos.- À Fundação de Apoio à Pesquisa do Estado da Paraíba (FAPESQ) e ao Conselho Nacional de Desenvolvimento Científico e Tecnológico (CNPq) pelo apoio financeiro. Ao CNPq pela concessão de bolsa de produtividade em pesquisa a Sérgio S. Azevedo.

\section{REFERÊNCIAS}

Alves C.J., Vasconcellos S.A., Camargo C.R.A. \& Morais Z.M. 1996. Influência dos fatores ambientais sobre a proporção de caprinos soro-reatores para a leptospirose em cinco centros de criação do Estado da Paraíba, Brasil. Arqs Inst. Biológico, São Paulo, 63(2):11-18.

Araújo Neto J.O. 2005. Isolamento de Leptospira spp. a partir do trato genital de ovelhas abatidas no matadouro público de Patos, Estado da Paraíba, Brasil. Monografia de Graduação em Medicina Veterinária, Universidade Federal de Campina Grande, Patos, Paraíba. 58p.

Brasil 2007. Sistema IBGE de recuperação automática (Sidra): pesquisa da pecuária municipal. Instituto Brasileiro de Geografia e Estatística (IBGE). Disponível em <http://www.sidra.ibge.gov.br/bda/tabela/listabl.asp?c=73\&z=t\&o=20> Acesso 30 mar. 2009.
Cole J.R., Sulzer C.R. \& Pursell A.R. 1973. Improved microtechnique for the leptospiral microscopic agglutination test. Appl. Microbiol. 25(6):976-980.

Cordeiro P.R.C. 1998. 0 desenvolvimento econômico da caprinocultura leiteira. Revta CFMV, Brasília, 4(13):28-30.

Corrêa M.O.A., Hyakutake S., Natale V., Tiriba A.C. \& Galvão P.A.A. 1964. Leptospiroses humanas ainda não assinaladas no Brasil. Revta Inst. Med. Trop. 6(2):71-74.

Cunha E.L.P., Mota R.A., Meireles L., Silva A.C.C., Silva A.V. \& Langoni H. 1999. Pesquisa de aglutininas anti-Leptospira em soros de caprinos no Estado de Pernambuco, Brasil. Revta Bras. Med. Vet. 21:38-40.

Dean A.G. 1994. Epiinfo version 6: a word-processing, database, and statistic program for public health on IBM-compatible microcomputers. Center for Diseases Control and Prevention, Atlanta. 601p.

Faine S., Adler B., Bolin C. \& Perolat P. 1999. Leptospira and leptospirosis. $2^{\text {nd }}$ ed. Medisci, Melbourne. 296p.

Galton M.M., Sulzer C.R., Santa Rosa C.A. \& Fields M.J. 1965. Application of a microtechnique to the agglutination test for leptospiral antibodies. Appl. Microbiol. 13(1):81-85.

Genovez M.E., Del Fava C., Castro V., Gregory L., Ferrari C.I.L., Lança Neto P., Souza M.R., Gotti T.B., Oliveira J.C.F. \& Pituco E.M. 2006. Effect of Leptospira spp. serovar hardjo infection on reproduction of two beef nelore herds with different serological status. World Buiatric Congress, France. 24p.

Herrmann G.P., Lage A.P., Moreira E.C., Haddad J.P.A., Resende J.R., Rodrigues R.O. \& Leite R.C. 2004. Soroprevalência de aglutininas anti-Letospira spp. em ovinos nas Mesorregiões Sudeste e Sudoeste do Estado do Rio Grande do Sul, Brasil. Ciência Rural 34(2):443-448.

Higino S.S.S. 2007. Isolamento de Leptospira spp. a partir do trato geniturinário de ovinos abatidos no matadouro público de Patos, Estado da Paraíba, Brasil. Monografia de Graduação em Medicina Veterinária, Universidade Federal de Campina Grande, Patos, PB. 44p.

Jordan D. 1996. Aggregate testing for the evaluation of Johne's disease herd status. Aust. Vet. J. 73(1):16-19.

Langoni H., Marinho M., Baldani S., Silva A.V., Cabral K.G. \& Silva E.D. 1995. Pesquisa de aglutininas anti-Leptospiras em soros ovinos do Estado de São Paulo, Brasil, utilizando provas de macroaglutinação em placa e soroaglutinação microscópica. Revta Bras. Med. Vet. 17(6):264-268.

Leon-Vizcaino L., Mendoza M.H. \& Garrido F. 1987. Incidence of abortions caused by leptospirosis in sheep and goats in Spain. Comp. Immunol. Microbiol. Infect. Dis. 10:149-153.

Lilenbaum W., Varges R., Medeiros L., Cordeiro A.G., Cavalcanti A., Souza G.N., Richtzenhain l.J. \& Vasconcellos S.A. 2008. Risk factors associated with leptospirosis in dairy goats under tropical conditions in Brazil. Res. Vet. Sci. 84(1):14-17.

Luiz R.R. \& Magnanini M.M.F. 2005. 0 tamanho da amostra em investigações epidemiológicas, p.295-307. In: Medronho R.A., Carvalho D.M., Bloch K.V., Luiz R.R., Werneck G.L. (Eds), Epidemiologia. Atheneu, São Paulo.

Martin S.W., Shoukri M. \& Thorburn M.A. 1992. Evaluating the health status of herds based on tests applied to individuals. Prev. Vet. Med. 14(1/2):33-43.

Noordhuizen J.P.T.M., Frankena K., Van Der Hoofd C.M. \& Graaf E.A.M. 1997. Application of quantitative methods in Veterinary Epidemiology. Wageningen Pers, Wageningen. 445p.

Schimidt V., Arosi A. \& Santos A.R. 2002. Levantamento sorológico da leptospirose em caprinos leiteiros no Rio Grande do Sul, Brasil. Ciência Rural 32(4):609-612.

Silva M.L.C.R., Pereira A.R.C. \& Neto J.O.A. 2006. Inquérito soro-epidemiológico para a leptospirose caprina nas microrregiões do Cariri Ocidental e Cariri Oriental do Estado da Paraíba, Brasil. Congresso Nordestino de Produção Animal, Petrolina.

Thrusfield M. 1995. Veterinary Epidemiology. $2^{\text {nd }}$ ed. Blackwell Science, Cambridge.

Vasconcellos S.A., Ohtsubo I., Yasuda P.H., Moretti A.S.A., Ito F.H., Passos E.C. \& Côrtes J.A. 1990. Efeito da concentração do soro sobre a sensibilidade e a especificidade da reação de soroaglutinação microscópica aplicada ao diagnóstico da leptospirose suína, tendo como antígeno a L. biflexa estirpe Buenos Aires. Braz. J. Vet. Res. Anim. Sci. 27(1):33-39. 